\title{
Coulisses
}

Revue de théâtre

\section{Quatre prologues pour Faust}

(et quelques personnages pour accueillir le public)

\section{François-Gildas Tual}

\section{OpenEdition}

\section{Journals}

Édition électronique

URL : https://journals.openedition.org/coulisses/299

DOI : 10.4000/coulisses.299

ISSN : 2546-9460

Éditeur

Presses universitaires de Franche-Comté

\section{Édition imprimée}

Date de publication : 31 décembre 2011

Pagination : 79-90

ISBN : 978-2-84867-404-9

ISSN : 1150-594X

Référence électronique

François-Gildas Tual, «Quatre prologues pour Faust », Coulisses [En ligne], 43 | Automne 2011, mis en ligne le 30 novembre 2016, consulté le 29 décembre 2022. URL : http://journals.openedition.org/ coulisses/299; DOI : https://doi.org/10.4000/coulisses.299 


\title{
Quatre prologues pour Faust
}

\author{
(et quelques personnages pour accueillir le public)
}

\author{
François-Gildas Tual
}

\author{
Il s'agissait de préparer un spectacle \\ qui poserait le problème du hasard, \\ et de l'intervention du public, \\ à partir du thème de Faust. \\ Michel Butor ${ }^{1}$
}

1 Dans une de ses nouvelles, Le Romancier Martin, Marcel Aymé raconte l'histoire d'un écrivain dont les personnages échappent au contrôle, et qui ne peut plus décider des trajectoires de son œuvre. Aussi étrange que paraisse le phénomène, nous pourrions nous poser la question suivante: le compositeur maîtrise-t-il sa création, ou l'œuvre, protéiforme, revendique-t-elle son autonomie pour être seule responsable de son évolution? Par association d'idées, l'artiste n'est-il pas un Faust aspirant à la connaissance mais incapable de saisir la véritable identité de l'ouvrage pourtant enfanté? Paul Valéry, dans son adresse "au lecteur de bonne foi et de mauvaise volonté ", à propos de "Mon Faust " (Ébauches), affirme que "rien ne démontre plus sûrement la puissance d'un créateur que l'infidélité ou l'insoumission de sa créature. Plus il l'a faite vivante, plus il l'a faite libre. $»^{2}$ Et de promettre aux héros de Goethe un bel avenir, loin des chemins précédemment tracés par Goethe...

Dans Votre Faust, Michel Butor et Henri Pousseur ont décrit un musicien, Henri, qui essaie de composer un opéra, un Faust. Il ne l'achèvera jamais, contraint d'abandonner ou acculé à la mort. Au centre de l'opéra, des spectacles de marionnettes avec Faust, Don Juan et Orphée: autant de héros entraînés par leurs désirs dans d'éprouvantes descentes aux enfers. Autant d'échecs qui reflètent surtout l'impuissance de l'artiste quand celui-ci prétend commander les processus de la création, à une époque où René Leibowitz affirme, dans son Introduction à la musique à douze sons, que toute série place l'œuvre, dès sa première ébauche, sous l'emprise du compositeur... Si quelques deux cents ans d'explorations musicales n'ont pas épuisé le mythe, sans doute le devonsnous tout autant à la multiplicité qu'à l'intemporalité du sujet. Selon Michel Butor, «le thème de Faust est très encombré; on ne peut donc pas se comporter comme si on était le premier à parler de ce personnage. $»^{3}$ Philippe Fénelon: « un thème comme celui de 
Faust est inépuisable, et l'on peut toujours en donner une nouvelle image. $»^{4}$ Pour l'homme d'aujourd'hui comme pour l'homme d'hier, "franchir les limites de la connaissance et $\mathrm{du}$ pouvoir humain pour pénétrer les mystères du monde est une aspiration insensée, qui se heurte au temps et à la finitude. $\rrbracket^{5}$ Explication parmi d'autres; chaque société, chaque individu a une bonne raison de puiser dans le mythe. Ajoutons à cela la diversité formelle des reprises, la multiplicité des sources littéraires et historiques, du personnage et de son devenir tant il est vrai que le seul Faust de Goethe se conjugue au pluriel, et il ne reste plus qu'à adapter l'histoire au genre choisi, adapter le genre à l'histoire qu'on veut écrire, se libérer des vieux modèles et expérimenter les formes hybrides suivant l'exemple offert par Goethe dans ses versions successives et plus ou moins fragmentaires.

3 Avec ou sans voix, sur scène ou au concert, Faust est un miroir, parfait symbole d'un insatiable appétit de liberté. Miroir positif ou négatif: en 1587, dans l'Histoire du docteur Johannes Faustus parue chez Spies à Francfort-sur-le-Main, un avertissement s'étonnait déjà que personne n'ait pensé à publier cette terrible histoire en tant que mise en garde pour la chrétienté, le « juste châtiment » de Faust pouvant inquiéter les « impies pleins d'orgueil et d'excessive curiosité. » Si les enjeux moraux ont vieilli, reconnaissons que le procédé n'a rien perdu de son efficacité; presque quatre cents ans plus tard, de nombreux opéras faustiens s'ouvrent sur de tels préambules où un personnage, héritier de quelques choreutes, parle directement au public. Que l'on pense seulement au Poète de Busoni (Doktor Faust, 1925), au Narrateur de Schnittke (Historia von D. Johann Fausten, 1995), à l'Homme de Philippe Fénelon (Faust, 2007), ou au Compositeur et au Directeur de théâtre, doubles très actuels de Faust et de Méphisto dans l'étrange Votre Faust de Michel Butor et Henri Pousseur $(1968)^{6}$. Certes, tous ne jouent pas le même rôle; tandis que le Poète encadre la représentation, le Narrateur demeure en marge du drame, l'Homme prend soudainement part à l'action, et le Compositeur est à la fois Henri Pousseur et, selon le prénom goethéen, Henri Faust. Mais en s'inscrivant dans une tradition où le théâtre informe le spectateur sur son propre monde, tous en disent long sur les nouvelles significations du mythe dans l'espace musical des $\mathrm{xx}^{\mathrm{e}}$ et $\mathrm{XxI}^{\mathrm{e}}$ siècles.

\section{Prologue 1 : le Poète}

Dans le Faust de Goethe déjà, entre la Dédicace et le Prologue dans le ciel, se trouvait le Prologue sur le théâtre avec une curieuse causerie du Directeur, du Poète et du Bouffon. Et de s'écrier avec le premier: «Ah! mon spectacle, à moi, c'est d'observer la foule, quand le long des poteaux elle se presse et roule... $»^{7}$ Le théâtre regarde avant d'être regardé, voit et donne à voir ce qui n'est pas vu. La foule? Le «tourbillon vulgaire et rongé par l'ennui » du public. La postérité? «Mot bien sublime! »Sans négliger «le devoir du poète et son emploi divin! » Loin de se contenter d'installer un décor ou des personnages, l'introduction discute de la pertinence et de la valeur de l'ouvrage. On songe aux monologues qui ont accompagné les débuts de l'opéra, manifestes prononcés par la Tragédie dans l'Eurydice de Peri, par la Musique dans l'Orfeo de Monteverdi, ou quelques années plus tôt par Lelio dans l'Amfiparnaso, comédie madrigalesque d'Orazio Vechi:

Illustres spectateurs, vous avez l'habitude de voir sur scène des tragédies, ou des comédies avec machines ornées de diverses manières, mais ce n'est pas une raison pour dédaigner notre comédie. Elle n'offre pas de scène riche ou gracieuse, mais présente une double nouveauté. La cité où se déroule l'action est le grand théâtre 
$\mathrm{du}$ monde, et c'est pour cela que tous veulent l'écouter. Mais sachez encore que le spectacle dont je parle s'apprécie seulement avec l'esprit; on y pénètre avec les oreilles et non avec les yeux. Par conséquent, faites silence et, au lieu de regarder, écoutez ${ }^{8}$.

Qui mieux que Faust peut alors porter une œuvre destinée à l'esprit, parlant d'ellemême avant de faire entrer ses personnages? Faust, explique Emmanuel Reibel, est « devenu un paradigme privilégié à partir duquel on dit la musique, on décrit sa trajectoire historique, on pense son rapport au passé. $»^{9}$ On pense évidemment à Adrian Leverkühn, compositeur visionnaire de Thomas Mann, mais aussi à ces prologues réfléchissant sur l'art. Ainsi le discours du Poète de Busoni, semblant compléter les propos précédents de Lelio:

De la vie, la scène reproduit les gestes, mais porte en son fronton, gravé, le mot: «contrefaçon»; afin qu'elle ne soit un modèle déformant, il faut donc que, magique, elle agisse comme un reflet honnête et beau; reconnaissez que, si elle écorne le vrai, à l'Incroyable elle sait rendre justice : la dit-on véridique, vous vous en gaussez, mais comme simple jeu, voici qu'elle vous rend graves et attentifs. Sous cette forme seule elle en appelle aux sons, car la musique se tient loin du commun; l'air est son corps, ses accents, le désir, et elle plane... Le merveilleux est son domaine ${ }^{10}$.

Renvoyant aux harangues du Dompteur de Lulu (Berg) et aux préparatifs d'Ariane à Naxos (Strauss), ce monologue est chargé d'histoire, et ses intentions théoriques pourraient faire de Doktor Faust une sorte de mise en application des principes esthétiques énoncés par Busoni dans un article publié dix ans plus tôt:

L'opéra du surnaturel, ou du non-naturel, serait le seul à s'octroyer le domaine des apparitions et des sentiments, et, ainsi, il construirait un monde d'apparences, qui reflèterait la vie dans un miroir magique ou un miroir humoristique; volontairement, il offrirait ce que le quotidien nous refuse. Le miroir magique pour l'opéra sérieux, et le miroir humoristique pour l'opéra bouffe. Entremêlons la danse, le jeu des masques, les apparitions, afin qu'à chaque instant le spectateur remarque le charmant mensonge pour ne pas le vivre comme une réalité.

Comparable à l'artiste qui ne peut être ému au moment où il doit émouvoir - car il se doit de garder toute la maîtrise de ses moyens -, le spectateur ne peut considérer l'action théâtrale comme une réalité, s'il veut l'apprécier; le plaisir artistique ne doit pas se laisser corrompre par le sentiment humain. L'acteur « joue »- il ne vit pas. Le spectateur demeure incrédule, et ainsi sa perception et son jugement restent libres ${ }^{11}$.

7 Pour Busoni, Faust force l'opéra à s'extraire du réel et à tourner le dos aux apparences du vérisme. Le sujet ayant préoccupé le compositeur pendant de longues années, devons-nous en conclure que celui-ci se dissimule sous le masque du héros ${ }^{12}$ ? Sans doute autant que sous le masque du Poète: « Depuis l'enfance, un conte me fascine où le

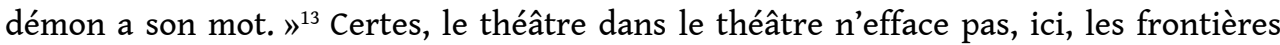
entre la scène et le public. Le Poète lui-même confirme que ce qui suit n'est qu'une histoire, issue des jeux de marionnettes plutôt que du Faust goethéen surexploité. Mais le spectateur, assuré de ne pas se confondre avec son propre reflet, n'en peut que mieux se contempler. Détachement synonyme de prise de conscience, annonçant le théâtre épique de Brecht et ses principes de distanciation; quelques décennies plus tard, le public de Votre Faust vivra une expérience forcément différente quand, invité à faire entendre sa voix, il croira avoir son mot à dire sur le déroulement du drame et sur son dénouement... 


\section{Prologue 2 : du Narrateur à l'Homme}

8 En fait, comme dans le Faust de Goethe et ses préambules successifs, il y a plus qu'un simple dédoublement de l'espace scénique et temporel dans le Faust de Busoni. Ce que nous avons nommé prologue n'était qu' «adresse du Poète aux spectateurs", monologue précédant deux autres prologues. De même que dans la conférence et les petites phrases émaillant le livret de Votre Faust, de même que dans certaines pages de l'opéra de Schnittke, la voix parlée semble rompre avec les conventions pour nous remmener dans le réel. Mise en abîme des niveaux du récit: du livre remis à Faust par les trois étudiants surgit la scène d'alchimie dans le même cabinet d'étude, et de l'invocation aux esprits, le retour de Faust dans le monde social, réalité qui pourrait n'être que fable ou rêve. Tout le fantastique dépend de ce subtil glissement vers l'Action principale, réinventant sans les couper les liens traditionnels de la causalité. Jamais représentée car publiée uniquement en tête de la partition pour chant et piano, la conclusion du Poète insiste, comme la préface de 1587, sur la fonction éducative de la pièce:

Durant cette soirée, une image de la nostalgie humaine s'est déroulée devant vos yeux par la magie des sons; la pièce a voulu vous rendre compte des maladresses et

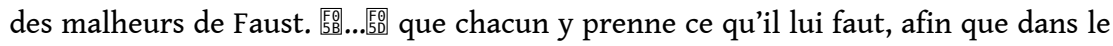
mouvement l'esprit s'ajoute à l'esprit : cela donnera un sens à l'ascension continue. La danse se refermera alors en un cercle parfait ${ }^{14}$.

9 De nouveau, on devine que le personnage principal pourrait-être à la fois Faust et le théâtre. Le contenu et la forme peut-être. Ou bien le sujet apparent et connu à côté de sa réinterprétation. Le "chacun" de Busoni, c'est le spectateur et le poète. La pièce doit être exemplaire d'un point de vue moral et d'un point de vue dramatique. Et parce que, trop souvent, «le public ne sait pas et ne veut pas savoir que, pour goûter une œuvre d'art, la moitié du travail doit être effectuée par celui qui la goûte $»^{15}$, le plus remarquable trait commun à nos opéras faustiens serait leur façon d'interpeller le spectateur. Que l'on pense, par exemple, à l'Historia von D. Johann Fausten de Schnittke, elle aussi inspirée par le Faust du seizième siècle et, sinon œuvre de toute une vie, résultat d'une longue élaboration qui a vu son projet initial remis en cause et modifié à maintes reprises. Pour Schnittke, Faust n'était pas «le pécheur marqué au fer rouge, définitivement condamné, car son destin (pas celui du personnage "physique", mais du personnage "moral " qui continue à vivre) n'est pas encore écrit jusqu'au bout et renferme des possibilités qui dépendent à leur tour de l'attitude que les hommes adopteront, dans la perspective posthume, à l'égard de sa destinée. $»^{16}$ Devenu Narrateur, le poète n'en intervient que plus souvent, dans le prologue tout d'abord où il n'hésite pas à informer le public sur le futur dénouement, puis de façon ponctuelle, parfois secondé par un chœur changeant régulièrement de costume. Chose fascinante que le partage du récit au début du troisième acte, quand Méphisto entreprend de raconter à son tour la mort de Faust, ou quand le Narrateur reprend la parole pour terminer l'histoire et, tourné vers le public, conclure la «leçon » sur un "épilogue » éloquent:

Soyez lucides et veillez, car votre adversaire, le diable, est comme un lion rugissant qui rôde, à la recherche de qui dévorer : Résistez-lui dans la fermeté de la foi ${ }^{17}$.

Ainsi encore le Faust d'après Lenau de Philippe Fénelon:

Une modification importante du texte de Lenau est ma décision de rajouter un personnage, celui de l'Homme, qui est un peu comme le voyeur de cette histoire. Ce 
n'est pas l'ombre d'un Faust, mais plutôt son positif. Il raconte le cheminement de Faust un peu comme le ferait un évangéliste, même si l'écriture n'a rien à voir avec celle des passions de Bach. C'est évidemment la voix même de l'écrivain. Mais cet homme prendra à la fin une dimension singulière et m'a permis d'envisager une fin qui ne soit pas tragique. Au dernier tableau, l'Homme prendra en effet l'identité de Görg et révèlera à Faust qu'il y a toujours un chemin pour approcher de la vérité, et que ce chemin est celui de l'amour de la vie terrestre. Et lui n'aura pas besoin d'un Méphisto pour le guider. Du moins jusqu'à nouvel ordre ${ }^{18}$.

11 L'Homme alter ego de Faust? Tel l'Évangéliste évoqué par le compositeur, il « commente tout d'abord ce qu'il voit, regarde ce qui se passe avec une certaine distance, voudrait peut-être participer mais ne peut rien faire, impuissant face aux agissements de ce son-héros. Incapable de pénétrer l'espace théâtral proprement dit, il semble condamné à demeurer “à la limite” de cet espace, n'a que sa voix pour exprimer son désarroi. Pourtant, il ne s'adresse pas non plus directement au public et, contrairement à Tonio dans Pagliacci, ne suggère pas que le drame puisse le concerner. ${ }^{19}$ Sa situation change néanmoins quand, gratifié d'un nouveau nom, il réapparaît sous les traits de Görg pour poursuivre l'action une fois l'opéra achevé:

Entré dans l'histoire, il devient le seul à pouvoir montrer à Faust le chemin de la Vérité. Et Faust ne l'entend toujours pas. Le septième tableau sera déjà une chute en soi, puisque l'Homme devenu Görg sera le nouveau Faust, et parce que le public comprendra alors que son tour aussi viendra... Dès lors, l'Homme n'est peut-être pas aussi anonyme qu'on le pense. Mais il est aussi une sorte de protecteur du monde, et nous invite peut-être à relativiser le pessimisme de l'opéra. Rappelonsnous que, pour Lenau, l'œuvre était une réponse à des problèmes personnels et sociaux. Déçu par son voyage en Amérique, celui qui n'a pas réussi à s'adapter à la rudesse du Nouveau Monde s'interroge sur cette liberté qu'il avait cru enfin trouver, désirant échapper à l'Autriche de Metternich. Pour nous, le drame demeure toujours actuel dans l'atemporalité du tragique de l'existence humaine. Et Görg - conscience de Faust - apporte la clé en faisant découvrir que la liberté désirée est en chacun de nous et qu'il est vain de la chercher ailleurs. Cette révélation de l'homme libre entraîne donc le rebelle vers sa destruction. Ses aventures douloureuses n'ont été que l'histoire d'un sursis ${ }^{20}$.

\section{Action principale : le public en scène}

Entrant ou sortant de scène sans que nous sachions bien par quelle porte il se faufile, parvenant ou non à influencer le destin de Faust, le protagoniste du prologue possède un peu des sortilèges de Méphisto, plus humain toutefois à l'image du serviteur Wagner, également double du héros, non pas condamné à suivre la même route mais devant trouver ses propres réponses aux mêmes questions. Pour Philippe Fénelon (comme pour Schnittke et Busoni), Faust pose la question de l'ambivalence et de l'obligation de choisir. "Le thème de l'opéra », précise le compositeur, « est la quête de la liberté dans le rapport de l'intellect avec la sensibilité. » Un questionnement si vaste qu'il peut s'appliquer à toutes les époques et à tous les contextes, jusqu'à nourrir le débat sur les rapports que doit entretenir le créateur avec sa création. Venons-en alors à Votre Faust: s'ouvrant sur la conférence d'un musicien, on s'y interroge naturellement sur la place du compositeur. Se détourner de la maîtrise illusoire de l'œuvre impose un acte d'humilité. En introduisant différentes références stylistiques grâce à la combinatoire sérielle, Henri Pousseur accepte du langage tout ce qui ne lui appartient pas en propre, c'est-à-dire travaille à partir d'un langage déjà largement stylisé. C'est pourquoi il reconnaît, tel Michel Butor dans le domaine littéraire, des objets naturels 
d'identification dans le langage, indépendants de toute conception systématique ou formelle. Dans son article Composer (avec) des identités culturelles, il écrit:

Il est difficile de nier ou même tout simplement d'ignorer que les faits sonores élémentaires, qu'ils soient intentionnels ou non, deviennent des signes parce qu'ils rencontrent et stimulent une fondamentale capacité d'identification ${ }^{21}$.

Votre Faust superpose donc plusieurs niveaux d'identification, du bruit-signal immédiatement intelligible à la référence complexe, citation musicale ou littéraire exigeant du spectateur une quasi-égalité culturelle avec l'auteur. Investie d'une fonction critique, la confrontation stylistique ne s'oppose pas aux sources du sérialisme. À l'encontre de la prétendue table rase de Darmstadt, elle invite à ne pas sous-estimer le passé2 en reprenant à son compte la leçon de Schoenberg, défendant une modernité redevable à la tradition; chaque référence s'inscrit dans une nouvelle unité tout en préservant son identité et son autonomie, jamais malmenées mais dotées de nouvelles propriétés d'adaptation. Chez Busoni, chez Schnittke et chez Fénelon, le paysage musical étant aussi parsemé de nombreuses allusions faustiennes ou non, nous n'en pouvons que mieux constater, avec Emmanuel Reibel, que «Traiter le sujet de Faust aujourd'hui revient donc peu ou prou à mettre en scène la mémoire. ${ }^{23}$

Développant un procédé déjà employé par Henri Pousseur dans une révision de Répons (1965), Votre Faust s'ouvre donc sur la diffusion d'une conférence enregistrée. Théâtre dans le théâtre: le Directeur entre sur scène et commande au musicien un opéra. Seule condition: que cela soit un Faust. Évidemment, nous sommes déjà dans la pièce, et la mise en abîme se poursuit lorsque les personnages entendent assister à leur tour à une représentation de marionnettes:

Schéma 1. Le théâtre dans le théâtre de Votre Faust

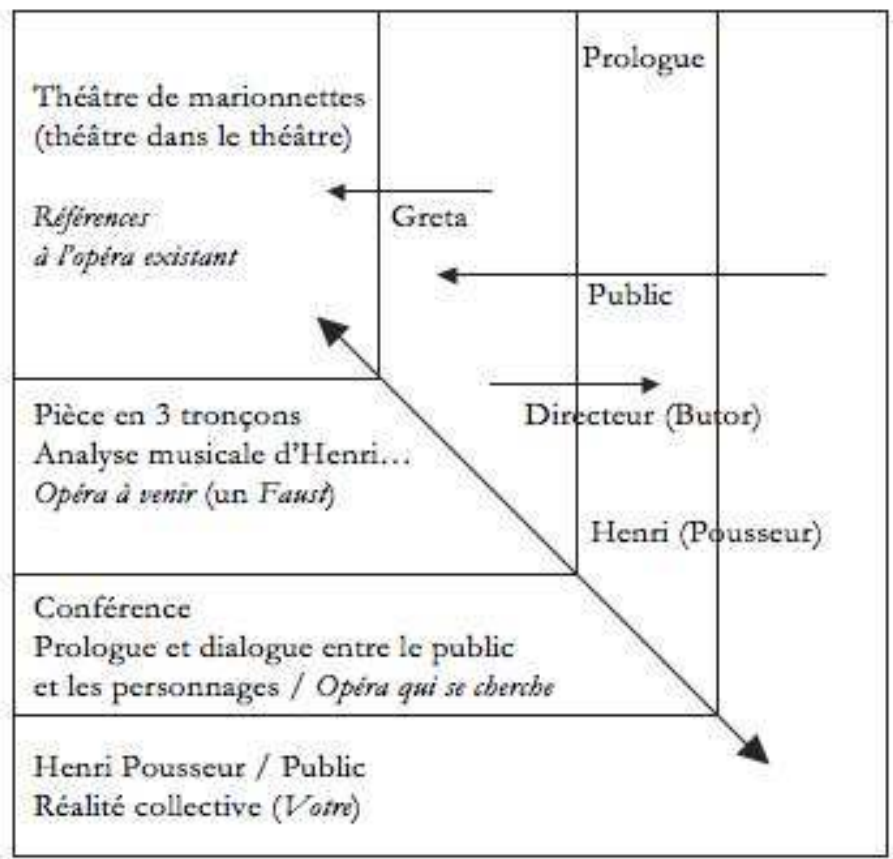

@François-Gildas Tual

Temps pédagogique préparant le public à l'opéra, la conférence est introduction à l'opéra et déjà dans l'opéra, soulignant l'intérêt de mieux associer le musicien et le 
public afin qu'il y ait compréhension mutuelle. Sans concession bien sûr. Souvenonsnous des recommandations de Busoni: "Même si l'art devait être complètement à la merci du public (ce que, pour ma part, je refuse), il faudrait maintenir une distance. Il descendrait au milieu du peuple et serait en même temps séparé de lui, comme il sied à un monarque. ${ }^{24} \mathrm{Au}$ compositeur la responsabilité de rendre son langage intelligible en considérant le niveau culturel de son auditoire; au public d'accepter d'être formé. Délivrer l'œuvre des exigences de son créateur implique les forces conjuguées de différents types de critiques. À celle des références s'ajoute l'entrée du public et de l'interprète. Si le style d'Henri Pousseur intègre les préoccupations prospectives et individuelles du musicien à partir d'un regard rétrospectif sur l'héritage collectif, elle repose aussi sur un renversement des données, par lequel le compositeur situe ses choix personnels dans l'élaboration, avant l'intervention du collectif en direct pendant l'exécution.

Dans Votre Faust, lorsque le public n'est pas satisfait par un dénouement, on revient en arrière pour prendre une nouvelle direction. La mobilité de la forme permet à l'œuvre de se corriger selon les attentes, et d'oublier les lois sérielles de l'irréversibilité au profit d'une poétique de l'imprévisibilité et de l'instant. L'issue n'est plus dictée par les choix initiaux du compositeur ou par un système musical logique et fermé, mais se dessine au fil des interventions extérieures en accord avec les alternatives prédéterminées. Compositeur, personnages, interprètes et spectateurs s'influencent mutuellement, mais chacun est seul maître et responsable de ses décisions. Il en résulte un réseau circulaire (et fermé) où chaque individu a un niveau différent de perception; le Directeur connaît les différents dénouements possibles mais essaie d'entraîner le public vers une fin négative, tandis que le public ne sait rien des futurs possibles mais tente de gagner la sortie la plus positive. Au centre, les interprètes obéissent plus ou moins docilement aux ordres...

Schémas 2 et 2 bis. Interactions dans Votre Faust
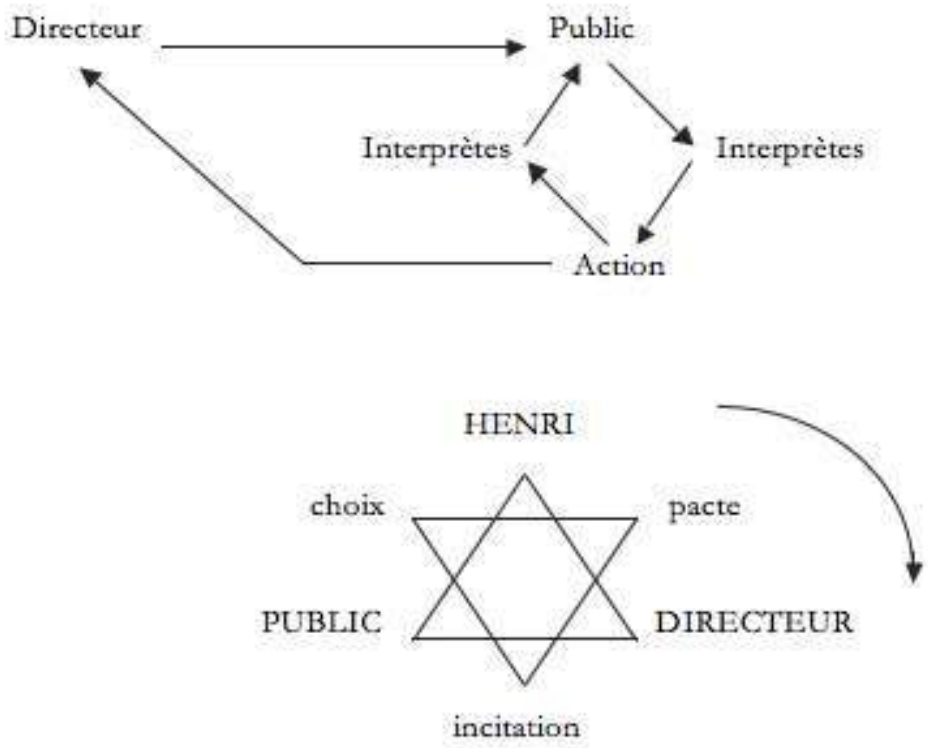

Interactions entre le directeur, le compositeur, le public et les interprètes.

@François-Gildas Tual 
Votre Faust est une œuvre ouverte. En insérant un maximum de données qui ne lui sont pas personnelles, Henri Pousseur perd le contrôle de sa création, réalisation inespérée du rêve d'Ernst Bloch de voir un jour le spectateur non pas "s'identifier purement et simplement » à ce qui se passe, mais de le voir « rester lucide » et, gardant ses distances avec le drame, dépasser le divertissement pour «apprendre en se recréant » ${ }^{25}$. De le voir surtout participer à l'action au point d'en interrompre le déroulement: « Peut-on dire "Cela est", quand tout ne fait que passer?» se demandait Werther... Et Faust: "Arrête-toi, tu es si beau». Reste que le public de Votre Faust ne découvre pas "l'instant suprême " puisque la pièce bientôt reprend. Mais tantôt passif, tantôt actif, à la fois passif et actif, le spectateur dialogue - a l'illusion de dialoguer-avec le compositeur. Extraordinaire réalisation scénique du schéma tripartite de la communication de Jean Molino ${ }^{26}$, Votre Faust non seulement rend perceptible la variabilité, mais propose aussi un nouveau réseau collectif de la création caractérisé par la réversibilité des relations:

Schéma 3. Le éseau collectif de création dans Votre Faust

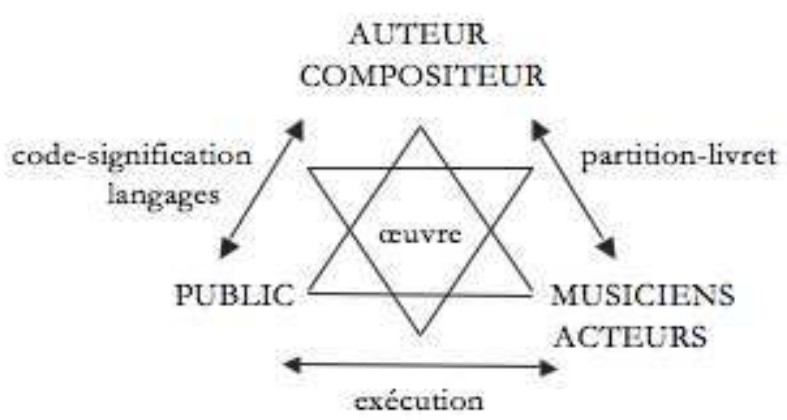

@François-Gildas Tual

Que reste-t-il alors au compositeur pour ne pas se faire, comme dans la nouvelle de Marcel Aymé, rejeter par son œuvre? Sans doute l'espace de satisfaction a-t-il évolué, et Jean-Yves Bosseur parle, à ce propos, d'écriture non pas d'« une œuvre, mais dans une œuvre $\aleph^{27}$. Votre Faust est l'histoire d'un opéra en devenir, d'une œuvre qui se forme progressivement sous les yeux du public, semblant sans cesse reposer la question du Commandeur à Don Juan - « Viendras-tu? » - celle du directeur à Henri - « Voulez-vous me composer un opéra? » Pendant l'entracte, au moment du vote, avec l'invocation aux esprits: «Veni, veni O Mephistophelis! » Dans chaque décor et dans chaque petite phrase apparemment anodine: "Tu viens, chéri!»; «Maman! - Quoi! - Viens! - La paix! ». Dans l'appel de Maggy malade, rapporté par son médecin, et dans l'hésitation à se rendre aux spectacles... Partout cette idée de "venir ». Il y a les formes positives, celles interrogatives - «Viendra-t-il? »-, les réponses négatives: « Il ne viendra pas.» Les variations sur le verbe accompagnent les décisions d'Henri et du public parce que l'un et l'autre composent simultanément leur œuvre. Avec, pour aboutissement, noncomposition dans le premier cas, fixation sur un disque ou sur une bande cinématographique dans le second. Une fixation qui, aussi réussie soit-elle, a peut-être trahi les grandes idées, et laissé à l'épilogue indécis de Busoni, voire à l'inachèvement ou au possible prolongement des opéras de Schnittke et de Fénelon, l'honneur d'avoir su affranchir l'œuvre de la possession despotique de son créateur... 


\section{Épilogue}

En 1969, Votre Faust est créé à la Piccola Scala de Milan dans un tel désordre qu'Henri Pousseur et Michel Butor ne peuvent que constater le désastre. Interprètes et spectateurs sont totalement perdus dans la mobilité de l'ouvrage. Les uns ont incriminé le manque de répétition, les autres l'incohérence du livret ou de la mise en scène. Ce jour-là, l'œuvre en devenir a échappé à ses créateurs et à son public. N'écoutant qu'ellemême, elle a eu un comportement scandaleux. "J'espère que nous aurons encore souvent l'occasion de vous entendre ", s'écriait le Directeur. "Merci, encore merci, et bravo! » Triste sort, l'histoire n'a pas exaucé les vœux formulés dans le prologue, n'a pas non plus tenu les promesses de l'épilogue. La réalité n'a pas rendu au théâtre la monnaie de sa pièce, a refusé de se faire miroir de la scène... Faut-il en déduire que l'ambition du compositeur a mené l'opéra à sa perte? Si une reprise dans de meilleures conditions permettrait, selon Henri Pousseur, de répondre, la beauté et l'originalité de la démarche demeurent tout entières dans cet échec scénique, y demeurent bien plus encore que dans les déceptions et les explications théoriques de l'auteur. Imaginer une œuvre singulière en la déchargeant d'une individualité trop embarrassante: n'était-ce pas là l'acte le plus individuel qu'Henri Pousseur pouvait imposer à Faust?

Henri : Mais cet opéra?

Le Directeur : Vous n'en avez jamais écrit une note...

\section{NOTES}

1. Michel Butor, « Collaboration Butor/Pousseur », Musique en Jeu, 4, 1971, p. 81.

2. Paul Valéry, «Mon Faust » (ébauches), repris dans les CEuvres complètes, édition de Jean Hytier, Gallimard, «Bibliothèque de la Pléiade », tome 2, 1960, p. 276.

3. Michel Butor, op. cit., p. 86.

4. Philippe Fénelon, « Un état de nostalgie », propos recueillis par Christophe Ghristi, Programme du Théâtre du Capitole, repris par l'Opéra National de Paris, 2010, p. 35.

5. Philippe Fénelon, Opéra Magazine, avril 2007, p. 29.

6. À cette liste, n'ajouterions-nous pas le Lecteur de L'Histoire du soldat, voire le personnage de Sly, étrangement passé d'un prologue shakespearien à l'opéra sans prologue de Pascal Dusapin? "J'ai eu beaucoup de plaisir à réinventer le rôle de Sly", confie le compositeur, "Sly est probablement mon personnage shakespearien préféré. C'est un vagabond ivrogne qui, lors du prologue de La Sauvage apprivoisée, s'écroule ivre mort dans un coin de la scène. [...] Il s'agit donc bien d'une mise en scène du "théâtre dans le théâtre" qui était déjà une convention du XvI siècle. Mais le plus intéressant est que, une fois la vraie pièce commencée, sly disparaîtra progressivement pour n'y plus revenir ! Je me suis donc toujours demandé où il avait pu passer! [...] Lorsque enfin Faustus daigne l'apercevoir, il lui demande: "D'où viens-tu?"; et sly de répondre par une phrase quelque peu agrémentée du Livre de Job: "Je cours le monde, je vague, je baguenaude". " "À propos d'une histoire de Faustus ", Faustus, The last night, Programme de l'opéra de Lyon, 2006, p. 127-128. 
7. Goethe, Faust, traduction de Gérard de Nerval, reprise dans le Théâtre complet, Gallimard, «Bibliothèque de la Pléiade ", 1951, p. 955.

8. Traduction de Colette Trabucci, Disque Harmonia mundi HMC 901461, 1993.

9. Emmanuel Reibel, Faust, la musique au défi du mythe, Fayard, 1998, p. 303.

10. Traduction de Philippe Godefroid, L'Avant-Scène Opéra, 193, novembre-décembre 1999, p. 17.

11. Ferruccio Busoni, « De l'avenir de l'opéra » (Von der Zukunft der Oper, Berlin 1913), L'Esthétique musicale, textes réunis par Pierre Michel et traduits par Daniel Dollé et Paul Masotta, Minerve, 1990, p. 89.

12. De Faust, on devine déjà la présence dans un conte fantastique rédigé par le musicien à l'âge de seize ans, où un pécheur signe un pacte avec le diable afin de s'emparer d'un trésor englouti.

13. L'Avant-Scène Opéra, 193, op. cit., p. 17.

14. Ibid., p. 75.

15. Ibid., p. 90.

16. Alfred Schnittke, «Über das Faust-Problem und über Peer Gynt », entretien avec Alexander Iwaschkin (1989), reproduit dans le programme de l'Opéra de Hambourg, mai 1996, p. 30.

17. Traduction de Jacques Fournier, Disque RCA 0902668413 2, 1996.

18. Philippe Fénelon, « Un état de nostalgie », op. cit., p. 35.

19. Philippe Fénelon, propos recueillis par nous-mêmes, avril 2011.

20. Idem.

21. Henri Pousseur, "Composer avec des identités culturelles ", InHarmoniques, 2, I.R.C.A.M./ Christian Bourgois Éditeur, 1987, p. 174.

22. Sur le retour aux sources, voir aussi le Procès du jeune chien où Michel Butor et Henri Pousseur, de nouveau réunis, racontent l'histoire d'un jeune compositeur confronté à ses propres origines.

23. Emmanuel Reibel, op. cit., p. 296.

24. Ferruccio Busoni, «Combien de temps encore" (Wie lange soll das gehen ?, 1910), L'Esthétique musicale, op. cit., p. 96.

25. Ernst Bloch, Le Principe Espérance (1959), traduction de Françoise Wuilmant, Gallimard, volume 1, 1976, p. 490. «Il est vrai que jusqu'à présent, aucune dramaturgie - et certainement pas celle dont les formes atteignent à la plus grande perfection - n'a témoigné d'une relation autonome de théorie à pratique, n'a présenté le drame comme un apprentissage susceptible de toujours se corriger (par l'irruption de tableaux). [...] N'a-t-il pas toujours existé des drames à plusieurs versions possibles, à plusieurs valorisations du parcours et du dénouement?»(ibid., p. 482)

26. Jean Molino, « Fait musical et sémiologie de la musique », Musique en jeu, n¹7, 1975, p. 37-62.

27. Jean-Yves Bosseur, Votre Faust, thèse de Doctorat de l'Université de Paris VIII, 1976, p. 129.

\section{INDEX}

oeuvrecitee Faust

Palavras-chave : O Seu Fausto, opera, intertextualidade, adaptação

Palabras claves : Su Fausto, opera, intertextualidad, adaptación

Keywords : Your Faust, opera, intertextuality, adaptation

Mots-clés : Votre Faust, opéra, intertextualité, adaptation 
AUTEURS

FRANÇOIS-GILDAS TUAL

Conservatoire à Rayonnement Régional de Grenoble 